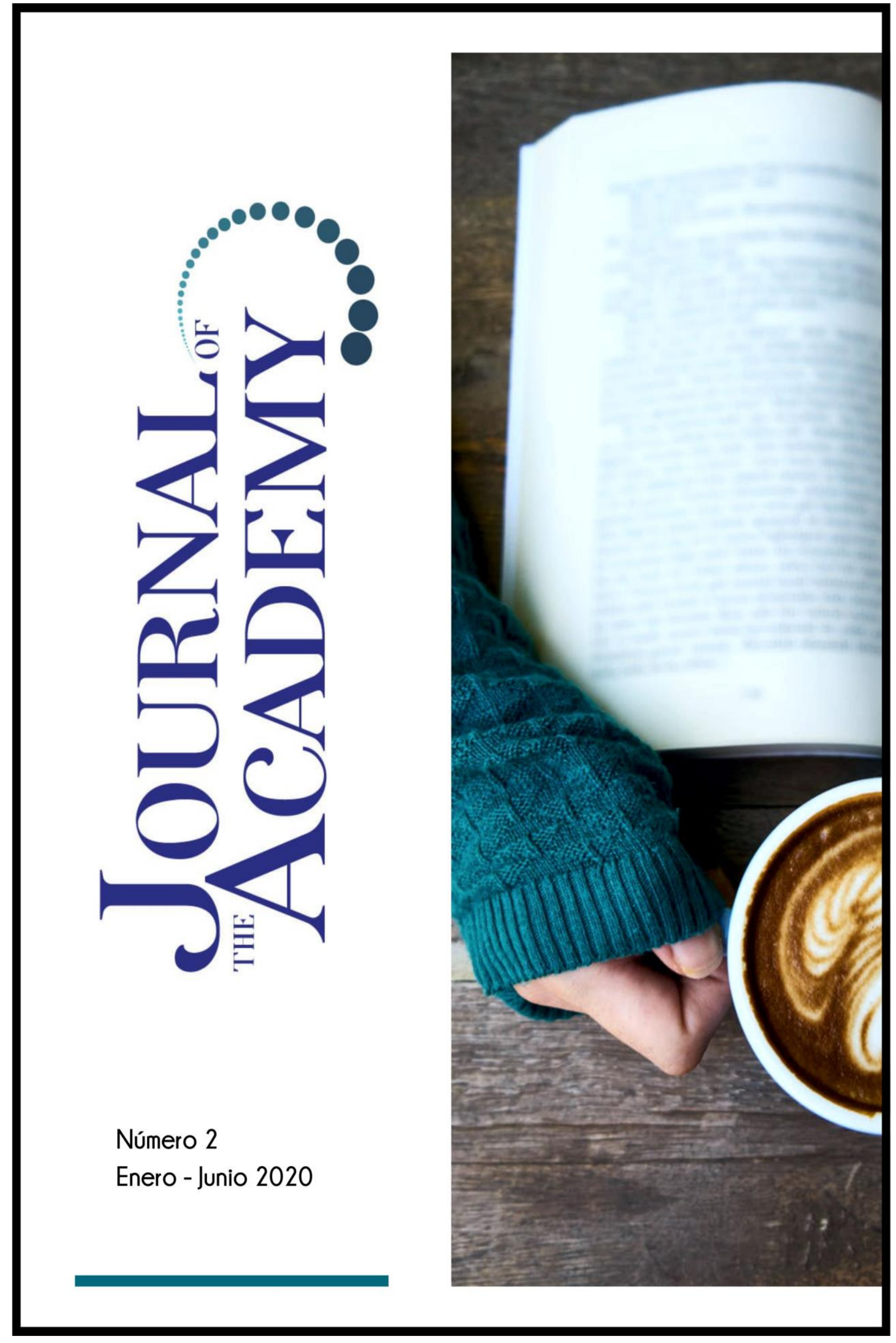




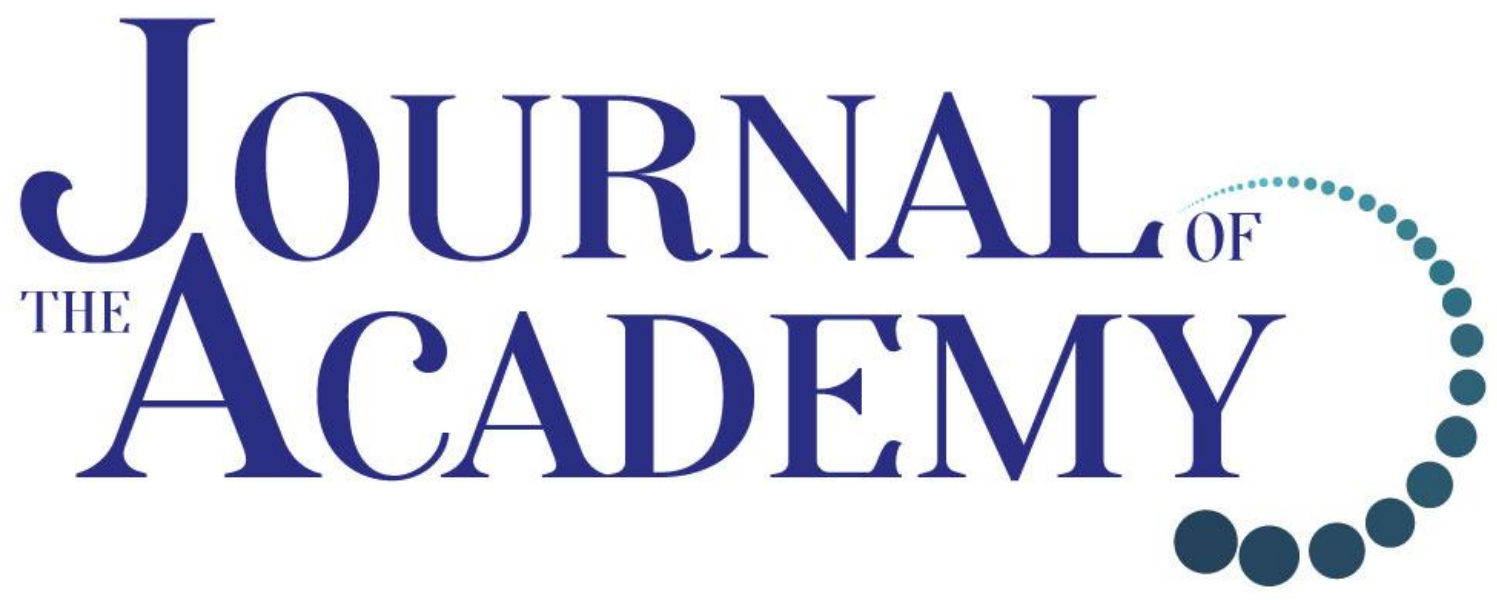

Publicación Científica de la Asociación de Universidades del Perú ASUP

Número 2

Enero-Junio 2020

www.journalacademy.org 


\section{ISSN 2707-0301}

\section{Directora-Editora en Jefe}

Ada Gallego Ruiz Conejo

Editor Científico

Oswaldo Orellana Manrique

Comité Científico

\section{Javier Carreón Guillén}

Universidad Nacional Autónoma de México, México Martino Contu

Universidad de Sassari, Italia

Jorge Enrique Elías Caro

Universidad del Magdalena, Colombia

Roberto Escalante Semerena

Universidad Nacional Autónoma de México, México

Oscar Ortega Arango

Universidad Autónoma de Yucatán, México

Alex Veliz Burgos

Universidad de Los Lagos, Chile

Comité Editorial

\section{Manuela Garau}

Centro Studi Sea, Italia

José Manuel González Freire

Universidad de Colima, México

Carlos Tulio da Silva Medeiros

Diálogos en Mercosur, Brasil

Eduardo Gomes Onofre

Universidade Estadual da Paraíba, Brasil

\section{Equipo Ejecutivo}

Juan Carlos Norabuena Castañeda

Thalia Chávez Cortéz

Journal Academy

Revista Semestral Open Access 


\title{
ASOCIATIVIDAD Y CRECIMIENTO ECONÓMICO DE PEQUEÑAS UNIDADES AGRÍCOLAS RURALES DE ANDAHUAYLAS, APURÍMAC
}

\section{ASSOCIATIVITY AND ECONOMIC GROWTH OF SMALL RURAL AGRICULTURAL UNITS OF ANDAHUAYLAS, APURÍMAC}

Recibido: 15 de julio de 2019

Aceptado: 23 de noviembre de 2019

Simón José CAMA FLORES

Universidad Nacional José María Arguedas, Perú

camasjf@hotmail.com

\begin{abstract}
Goals. It is to determine the relationship that exists between associativity as a management model and the economic growth of small rural agricultural units in the province of Andahuaylas. Materials and methods. An observational, analytical cross- sectional investigation was carried out in the months of October to December 2017 in the producer associations of the districts of Huancabamba, Pacucha, Andahuaylas, Talavera and Turpo. As a population, the members of the 5 associations were included and intentional non-probabilistic sampling was carried out, consisting of 109 associated potato and quinoa producers, who were previously informed of the objectives of the study. As for the instrument, a questionnaire was elaborated with 35 items divided into two sections, the survey was anonymous and based on face-to-face interviews.
\end{abstract}

Keywords: Associativity; Economic growth; Agricultural units

Resumen: Objetivos. Es determinar la relación que existe entre la asociatividad como modelo de gestión y el crecimiento económico de las pequeñas unidades agrícolas rurales de la provincia de Andahuaylas. Materiales y métodos. Se realizó una investigación correlacional, de corte transversal, en las asociaciones de productores de los distritos de Huancabamba, Pacucha, Andahuaylas, Talavera y Turpo. Como población se incluyó a 5 asociaciones y se aplicó muestreo no probabilístico intencional y conformado por 109 productores asociados de papa y quinua. En cuanto al instrumento se elaboró un cuestionario con 35 ítems dividido en dos secciones, la encuesta fue anónima y basadas en entrevistas cara a cara.

Se ha aplicado la confiabilidad alfa de Cronbach la consistencia del instrumente y ha sido aplicado una encuesta piloto a 22 asociados, se tuvo el siguiente índice de correlación igual a 0,852, el instrumento tiene alta confiabilidad aceptable.

Palabras claves: Asociatividad; Crecimiento económico; Unidades agrícolas

\section{Para Citar este Artículo:}

Cama Flores, Simón José. Asociatividad y crecimiento económico de pequeñas unidades agrícolas rurales de Andahuaylas, Apurímac. Journal of Academy num 1 (2019): 01-10.

Licencia Creative Commons Atributtion Nom-Comercial 3.0 Uunported

(CC BY-NC 3.0)

Licencia Internacional

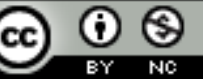


Journal of the Academy Número 2 Enero-Junio 2020 - Pag. 02

\section{Introducción}

La globalización ha generado grandes transformaciones en todo el mundo, en el aspecto económico, político, social, cultural, educativo, tecnológico. Los negocios, empresas, los productores la única forma de sobrevivir es asociándose, uniéndose, esta forma de trabajar es un modelo o estrategia de trabajo colectivo para conseguir beneficios colectivos, para cumplir con lo que el mercado exige calidad, variedad, cantidad por si solos difícilmente podemos cumplir, pero asociado se puede lograr muchos beneficios como reducir costos, acceso a mercados, financiamiento, buenos precios en la venta y compra. Crecimiento económico que se considera como proceso de mejora en calidad de vida de los productores agrícolas, alcanzar determinadas ganancias y éxito. Para los asociados existen ciertas carencias como la falta de información, capacitación, asistencia técnica, falta de capacidad de organización, apoyo financiero, altos costos de producción, restricciones en la comercialización de sus productos, falta desarrollar tecnología para mejorar la calidad de sus productos, por otro lado hay cierto desinterés en las actividades de cooperación agrícola y participación en acciones de interés común; así mismo, cuando compran enfrentan problemas del elevado precio de los insumos agrícolas, servicios y alquiler de maquinarias, entre otros. El objetivo de la investigación es determinar la relación que existe entre la asociatividad como modelo de gestión y el crecimiento económico de las pequeñas unidades agrícolas rurales de la provincia de Andahuaylas. La hipótesis es la Asociatividad como modelo de gestión se relaciona directamente con el crecimiento económico de las pequeñas unidades agrícolas rurales de la provincia de Andahuaylas

\section{Desarrollo}

\section{Materiales y métodos}

\section{Diseño y lugar de estudio}

Se realizó una investigación observacional, analítico de corte transversal en los meses de octubre, noviembre y diciembre de 2017 en las asociaciones de productores agropecuarios de Sumaq de Huinchos, Manchaybamba, Kuracallayocc, Waqoto Pampamarca y Jesús de buen pastor de Taypicha ubicados en los distritos de Huancabamba, Pacucha, Andahuaylas, Talavera y Turpo en la provincia de Andahuaylas Apurímac.

\section{Población}

Se incluyó a todos los integrantes de las 5 asociaciones y como muestra no probabilística intencional y está conformado por un total de 109 productores de papa y quinua, a los cuales se les informo previamente los objetivos del estudio. Los participantes fueron ubicados en los distritos donde están ubicados las asociaciones y en sus casas en horas de la mañana (7.00 am) y en la tarde $(5.00 \mathrm{pm})$ Los encuestadores que apoyaron fueron estudiantes universitarios, quienes estuvieron presentes durante todo el tiempo que tomo el llenado de la encuesta (10 a 15 minutos). Fueron excluidos los productores no asociados. El estudio fue aprobado por la dirección general de investigación e innovación de la Universidad Nacional José María Arguedas.

\section{Instrumento}

Se elaboró un cuestionario con 35 ítems dividido en dos secciones, la encuesta fue anónima y basadas en entrevistas cara a cara. En sección uno se diseñó 10 preguntas de datos generales, los cuales incluyeron género, nivel de estudios, beneficios entre otros y en la sección dos se elaboró 25 preguntas relacionados con la variable asociatividad y crecimiento económico. La cuál ha sido previamente validada por juicio de expertos (03 expertos) y se ha aplicado la confiabilidad alfa de Cronbach que mide la fiabilidad y 
consistencia del instrumente, para lo cual se ha diseñado un cuestionario compuesto por 25 ítems y ha sido aplicado mediante una encuesta piloto a 22 productores asociados, se tuvo el siguiente índice de correlación igual a 0,852 , el instrumento tiene alta confiabilidad aceptable, por lo tanto, es aplicable.

\section{Definición de variables}

Variable 1: Asociatividadç

Es la acción de cooperación entre los agricultores individuales y/o empresas agrícolas pequeñas y medianas que se unen por voluntad propia en un esfuerzo conjunto para enfrentar la globalización y competitividad de los mercados logrando un objetivo común (Ministerio de agricultura - unidad de agronegocios).

Dimensiones:

- Capacitación y tecnificación

- Reducción de costos

- Mayor poder de negociación

- Incremento de productividad

- Mejora organizacional

- Comercialización

\section{Variable 2: Crecimiento Económico}

Vázquez (2012) nos dice que "el crecimiento económico permite generar los recursos para incrementar el desarrollo humano, en tanto que el desarrollo humano, al mejorar el capital humano, incrementa la productividad y las posibilidades de crecimiento económico" (p. 9).

Dimensiones:

- Bienestar económico

\section{Análisis de datos}

Los resultados de las encuestas fueron digitalizados en Excel, todos los cuestionarios fueron llenados en forma completa los items. Se utilizó la prueba de chi-cuadrado de Pearson, para evaluar la relación o asociación entre la asociatividad como modelo de gestión y el crecimiento económico de las pequeñas unidades agrícolas rurales.

Como la probabilidad de $(p=0,000)$ es menor que el nivel de significancia $(\alpha=0,05)(p=0,000<\alpha=0.05)$, rechazamos la hipótesis nula y aceptamos la hipótesis alterna, es decir la Asociatividad como modelo de gestión se relaciona significativamente con el crecimiento económico de las pequeñas unidades agrícolas rurales de la provincia de Andahuaylas, a un nivel de confianza del 95\%. En el análisis de datos se realizó usando el programa estadístico SPSS v22.

\section{Resultados}

Se tomó en cuenta 5 asociaciones de productores tanto de papa y quinua, que conforman un total de 109 productores asociados, se encuesto a la totalidad. 


\section{Características de la población}

Tabla 01

Asociatividad como modelo de gestión y su relación con el crecimiento económico de las pequeñas unidades agrícolas rurales de la provincia de Andahuaylas, Apurímac

$-2017$

\begin{tabular}{|c|c|c|}
\hline Características generales & $\mathbf{n}$ & $\%$ \\
\hline \multicolumn{3}{|l|}{ Genero } \\
\hline$\checkmark \quad$ Femenino & 31 & 28,4 \\
\hline$\checkmark \quad$ Masculino & 78 & 71,6 \\
\hline \multicolumn{3}{|l|}{ Estudios alcanzados } \\
\hline$\checkmark \quad$ Primaria & 60 & 55,0 \\
\hline$\checkmark$ Secundaria & 45 & 41,3 \\
\hline$\checkmark$ Instituto tecnológico & 2 & 1,8 \\
\hline$\checkmark \quad$ Universidad & 2 & 1,8 \\
\hline
\end{tabular}

\section{Trabajando asociados obtendrá más beneficios}

\begin{tabular}{llll}
\hline$\checkmark$ Si & 103 & 94,5 \\
$\checkmark$ No & 6 & 5,5 \\
\hline
\end{tabular}

\section{Avance en el crecimiento económico}

\begin{tabular}{llll}
\hline$\checkmark$ & Regular & 80 & 73,4 \\
\hline$\checkmark$ & Bien & 25 & 22,9 \\
$\checkmark$ & Muy bien & 4 & 3,7 \\
\hline
\end{tabular}

Se arrepiente de haberse asociado

\begin{tabular}{cccc}
\hline & Si & 18 & 16,5 \\
$\checkmark$ & No & 91 & 83,5 \\
\hline \multicolumn{2}{l}{ La asociación cuenta con máquinas y equipos agrícolas } & \\
\hline$\checkmark \quad \mathrm{Si}$ & 99 & 90,8 \\
$\checkmark$ & No & 10 & 9,2 \\
\end{tabular}




\begin{tabular}{|c|c|c|}
\hline \multicolumn{3}{|c|}{ La asociación le apoya en la compra de insumos } \\
\hline$\checkmark \mathrm{Si}$ & 81 & 74,3 \\
\hline$\checkmark$ No & 28 & 25,7 \\
\hline \multicolumn{3}{|c|}{ Al haberte asociado ha aumentado tu producción } \\
\hline$\checkmark \mathrm{Si}$ & 94 & 86,2 \\
\hline$\checkmark$ No & 15 & 13,8 \\
\hline \multicolumn{3}{|c|}{ Al asociarte ha aumentado la venta de tu producto } \\
\hline$\checkmark \mathrm{Si}$ & 81 & 74,3 \\
\hline$\checkmark$ No & 28 & 25,7 \\
\hline
\end{tabular}

Fuente: elaboración propia usando SPSS v22

En la tabla 01 se observa que el $71,6 \%$ los asociados son de género masculino; el $55 \%$ de los asociados su nivel de estudio es primaria y $41,3 \%$ secundaria; el $94,5 \%$ están de acuerdo que asociados obtendrá más beneficios; en cuanto al crecimiento económico el 73,4 \% de asociados indicaron "Regular"; el 83,5 $\%$ no se arrepiente de haberse asociado; el 90,8 \% indican que su asociación cuenta con máquinas y equipos agrícolas; el 74,5\% indican que su asociación le apoya en la compra de insumos; el 86,2 \% de asociados manifiestan haber aumentado la producción de su producto; el 74,3 \% indican que al haberse asociado ha aumentado la venta de su producto.

\section{Asociatividad y Crecimiento Económico}

Tabla 02

Relación entre la Asociatividad como modelo de gestión y el crecimiento económico de las pequeñas unidades agrícolas rurales de la provincia de Andahuaylas, Apurímac - 2017

\begin{tabular}{lll}
\hline Asociatividad & \multicolumn{2}{l}{ Crecimiento económico } \\
\cline { 2 - 3 } & $\mathbf{n}$ & $\mathbf{p}$-valor \\
\hline Capacitación y tecnificación & 109 & 0,000 \\
Reducción de costos & 109 & 0,035 \\
Mayor poder de negociación & 109 & 0,004 \\
Incremento de productividad & 109 & 0,000 \\
Mejora organizacional & 109 & 0,000 \\
Comercialización & 109 & 0,006 \\
Asociatividad & 109 & 0,000 \\
\hline
\end{tabular}

Fuente: elaboración propia usando SPSS v22

Se hace un análisis de relación o asociación a través de chi-cuadrado de Pearson (Tabla 02), donde indica una relación directa y significativa para las variables Asociatividad como modelo de gestión y el crecimiento económico de las pequeñas unidades agrícolas rurales $(p=0,000)$. En cuanto a la relación de las dimensiones de la variable asociatividad y el crecimiento económico; se observa con la dimensión capacitación y tecnificación una relación directa y significativa $(\mathrm{p}=0,000)$; con la dimensión reducción de costos una relación directa y significativa $(p=0,035)$; con la dimensión mayor poder de negociación una relación directa y significativa $(p=0,004)$; con la dimensión incremento de productividad se observa una 
relación directa y significativa $(p=0,000)$; con la dimensión mejora organizacional se observa una relación directa y significativa $(p=0,000)$; con la dimensión comercialización se observa una relación directa y significativa $(p=0,006)$.

\section{Discusión}

A partir de la información obtenida, se relacionó la asociatividad con el crecimiento económico de las pequeñas unidades agrícolas rurales de la provincia de Andahuaylas. Se obtuvo un P-valor $=0,000$, como el nivel de significancia es menor que $0,05(p=0,000<\alpha=0.05)$ se rechaza la hipótesis nula, porque está altamente relacionada. Podemos afirmar que, con la asociatividad, se puede lograr muchos beneficios entre ellos el crecimiento económico, apoyo del gobierno y ONGs. Este resultado guarda relación con lo que sostienen Rojas, F. (2013) genera intervención del gobierno, mayor impacto económico; Ariza, O. \& Franco, E (2009) optimista por el modelo de asociatividad; Orrala, H. A. (2013) da importancia de que genera el desarrollo socio económico; Flores, K. (2010) se mantienen unidos los productores por un interés económico.

Se relacionó capacitación y tecnificación con el crecimiento económico de las pequeñas unidades agrícolas rurales. Se obtuvo un P- valor $=0,000$, como el nivel de significancia es menor que 0,05 $(p=0,000<\alpha=0.05)$, se rechaza la hipótesis nula, porque está altamente relacionada. De acuerdo a este resultado, podemos afirmar que la capacitación y tecnificación, que significa desarrollar, actualizar conocimientos, habilidades, capacidades en la gestión, manejo de herramientas y mejorar el proceso productivo para obtener productos de calidad, de esa manera comercializar mejor sus productos y esto conlleva al crecimiento económico de los productores. Este resultado guarda relación con lo que sostienen Rojas, F. (2013) desarrollo de la capacitación para los productores y Ariza, O. \& Franco, E (2009) acrecentar la labor de capacitación.

Se relacionó reducción de costos con el crecimiento económico de las pequeñas unidades agrícolas rurales. Se obtuvo un $P$ - valor $=0,035$, como el nivel de significancia es menor que $0,05(p=0,035<\alpha=0.05)$, se rechaza hipótesis nula, porque están altamente relacionadas. De acuerdo a este resultado, podemos afirmar que la reducción de costos en el proceso productivo agrícola, es decir en la utilización al máximo de los recursos (agua, suelos, lluvias, mano de obra, maquinas, financiero), se busca la compra y obtención de productos, insumos, tecnologías a costos más bajos, estos aspectos en asociatividad son beneficioso y este tiene relación con el crecimiento económico de los productores. Este resultado guarda relación con lo que sostienen Rojas, F. (2013) es más económico comprar en asociación que comprar individualmente y Flores, L. D. (2013) mejorando el acceso a tecnologías de productos o procesos y a financiamiento, a la vez que se comparte riesgos, costos y beneficios.

Se relacionó mayor poder de negociación con el crecimiento económico de las pequeñas unidades agrícolas rurales. Se obtuvo un P- valor $=0,004$, como el nivel de significancia es menor que 0,05 $(p=0,004<\alpha=0.05)$, se rechaza la hipótesis nula, porque están altamente relacionadas. En la actividad agrícola se tiene que negociar con varios grupos de interés como con proveedores, compradores, gobierno, otras organizaciones, negociar individualmente no es conveniente, si no que en colectivo es lo más conveniente para vender, comprar productos y recursos. Este resultado guarda relación con lo que sostiene Maldonado, G. M. (2012) principales impactos directos de la asociatividad, se identifica el acceso a nuevos mercados, el mayor poder de negociación y las mejoras en la gestión productiva.

Se relacionó incremento de productividad con el crecimiento económico de las pequeñas unidades agrícolas rurales. Se obtuvo un P- valor $=0,000$, como el nivel de significancia es menor que 0,05 $(p=0,000<\alpha=0.05)$, se rechaza la hipótesis nula, porque están altamente relacionadas. De acuerdo a la información aumenta la producción de los productores, pero el problema es que sus productos son de baja calidad, porque sus tierras están contaminadas con químicos, son pocos las tierras consideradas 
orgánicas, tienen mayor producción, pero sus productos son de baja calidad por tanto no son competitivos en el mercado. Este resultado guardan relación con lo que sostienen Rojas, F. con la asociatividad se han impulsado, generando así un mejoramiento en la competitividad que estas pueden tener en el mercado; Ariza, O. \& Franco E. se muestran optimistas por el modelo de la asociatividad, evitando con este proceso que las pequeñas y medianas empresas se salgan del mercado por falta de productividad y competitividad y Flores, L. D. implementación de un trabajo organizado en diversas actividades productivas, puede beneficiar en aprovechar las oportunidades del entorno, incrementando la producción y productividad.

Se relacionó mejora organizacional con el crecimiento económico de las pequeñas unidades agrícolas rurales. Se obtuvo un $\mathrm{P}$ - valor $=0,000$, como el nivel de significancia es menor que $0,05(p=0,000<\alpha=0.05)$, se rechaza la hipótesis nula, porque están altamente relacionadas. La gestión y control de los procesos de las organizaciones agrícolas como practicar la democracia en elección de sus autoridades, liderazgo, organizar las tareas, comunicación interna y externa, estructura organizacional. Este resultado guardan relación con lo que sostienen Maldonado, G.M. explica su auge por su modelo organizativo, en el cual se reconocen como factores de éxito los siguientes: capital social, el liderazgo, la transparencia, mecanismos de control, la flexibilidad, descentralización del poder, funciones, democracia en la toma de decisiones; Flores, K. una asociación desarrollada formalmente, con estructuras orgánicas establecidas, democracia entre sus miembros y objetivos comunes interiorizados enfocados hacia el crecimiento y Flores, L. D. el proceso de asociatividad depende en gran medida del grado de capital social existente entre los potenciales participantes, haciendo hincapié en la confianza, reciprocidad, compromiso, cooperación, fortalecer la parte organizativa, entre otros.

Se relacionó comercialización con el crecimiento económico de las pequeñas unidades agrícolas rurales. Se obtuvo un $\mathrm{P}$ - valor $=0,006$, como el nivel de significancia es menor que $0,05 \quad(p=0,006<\alpha=0.05)$, se rechaza la hipótesis nula, porque están altamente relacionadas. Es fundamental en una organización productiva, porque lo que produce tiene que vender al consumidor, la comercialización se relaciona con el crecimiento económico de las unidades productivas, si no hay ventas no habrá ganancias entonces no podremos satisfacer las necesidades vitales y mejorar la calidad de vida de los asociados. Este resultado guardan relación con lo que sostienen Ariza, O. \& Franco, E. una mentalidad abierta a los cambios que trae consigo la globalización de los mercados; Orrala, H. A. las estrategias propuestas dentro del presente estudio, garantizan la obtención de resultados de todo grupo asociativo, es decir, mejorara la rentabilidad aumentando las ventas de los productos agrícolas y Maldonado, G. M. los nichos de mercados especiales surgen por las tendencia actual de las exigencias del consumidor.

\section{Conclusiones}

Las conclusiones a que se llegó al finalizar esta investigación son las siguientes:

1. En esta investigación concluimos que si existe una relación entre la Asociatividad como modelo de gestión y el crecimiento económico de las pequeñas unidades agrícolas rurales de la provincia de Andahuaylas. Porque en la prueba de hipótesis de estudio se encuentra que el valor de probabilidad $(p=0,000)$ es menor que el nivel de significancia $(\alpha=0,05)$, por consiguiente, se acepta la hipótesis alternativa, a un nivel de confianza del 95\%.

2. Como resultado de la investigación presentada, podemos concluir que existe una relación entre la capacitación y tecnificación, y el crecimiento económico de las pequeñas unidades agrícolas rurales de la Provincia de Andahuaylas. Porque en la prueba de hipótesis de estudio 1 , se halla que $(p=0,000) p$ - valúe $<\alpha=0,05$; entonces se acepta la hipótesis alterna y se rechaza la hipótesis nula, al $95 \%$ de nivel de confianza. 
3. Como resultado de la investigación presentada, podemos concluir que existe una relación entre la reducción de costos y el crecimiento económico de las pequeñas unidades agrícolas rurales de la Provincia de Andahuaylas. Porque en la prueba de hipótesis de estudio 2, se halla que $(p=0,035) p$-valúe $<\alpha=0,05$; entonces se acepta la hipótesis alterna, al $95 \%$ de nivel de confianza.

4. Como resultado de la investigación presentada, podemos concluir que existe una relación directa y significativa entre el mayor poder de negociación y el crecimiento económico de las pequeñas unidades agrícolas rurales de la Provincia de Andahuaylas. Porque en la prueba de hipótesis de estudio 3 , se halla que $(p=0,004) p$ - valúe $<\alpha=0,05$; entonces se acepta la hipótesis alternativa y se rechaza la hipótesis nula, al $95 \%$ de nivel de confianza.

5. Como resultado de la investigación presentada, podemos concluir que existe una relación directa y significativa entre el incremento de la productividad y el crecimiento económico de las pequeñas unidades agrícolas rurales de la Provincia de Andahuaylas. Porque en la prueba de hipótesis de trabajo 4 , se halla que $(p=0,000) p$ - valúe $<\alpha=0,05$; entonces se acepta la hipótesis alternativa y se rechaza la hipótesis nula, a un nivel de confianza del $95 \%$.

6. Como resultado de la investigación presentada, podemos concluir que existe una relación directa y significativa entre la mejora organizacional y el crecimiento económico de las pequeñas unidades agrícolas rurales de la Provincia de Andahuaylas. Porque en la prueba de hipótesis de trabajo 5 , se halla que $(p=0,000) p$ - valúe $<\alpha=0,05$; entonces se acepta la hipótesis alterna y se rechaza la hipótesis nula, al 95\% de nivel de confianza.

Como resultado de la investigación presentada, podemos concluir que existe una relación directa y significativa entre la comercialización y el crecimiento económico de las pequeñas unidades agrícolas rurales de la Provincia de Andahuaylas. Porque en la prueba de hipótesis de trabajo 6 , se halla que ( $p=$ $0,006) p$ - valúe $<\alpha=0,05$; entonces se acepta la hipótesis alterna y se rechaza la hipótesis nula, al $95 \%$ de nivel de confianza.

\section{Referencias bibliográficas}

Amézaga, C., Rodríguez, D., Núñez, M. \& Herrera, D. (2013). Orientaciones Estratégicas para el Fortalecimiento de la Gestión Asociativa. El Salvador: Instituto Interamericano de Cooperación para la Agricultura (IICA). Esta publicación también está disponible en formato electrónico (PDF) en el sitio Web institucional en http://www.iica.int

Arias, F. (2012). El Proyecto de Investigacion, Introduccion a la Investigacion Cientifica (6a ed.). Caracas, Republica Bolivariana de Venezuela: Episteme, C.A.

Ariza, O. \& Franco, E. (2009) propuesta de asociatividad para el sector agropecuario de la provincia de Velez (Santander) como estrategia para generar competitividad (Tesis). http://gidrot.com/publicaciones/tesis/posgrado/2010-2009.html

Bada, L.; Rivas, L. \& Littlewood, H. (2017) Modelo de asociatividad en la cadena productiva en las Mipymes agroindustriales, (Revista científica), Universidad Nacional Autónoma de México, https://reader.elsevier.com/reader/sd/8B1CADB5A572AD519CB991A488D8C5F16D51D432C87BCB38A 84431E45F3AAD52224236FE98569011F98454D8D2F77FA7

Carrasco, S. (2017). Metodología de la investigación científica, editorial san marcos E.I.R. LTDA, cuarta edición. 
Chong Chong, Mario, (2012) Diseño de un modelo de gestión para el desarrollo de las pequeñas unidades agrícolas rurales del Perú: Talleres de asociatividad. Industrial Data [en linea] 2012, 15 (Enero-Junio): [Fecha de consulta: 18 de enero de 2018] Disponible <http://www.redalyc.org/articulo.oa?id=81624969005> ISSN 1560-9146

Daft, R. (2011) Teoría y diseño organizacional, impreso en México, décima edición, Cengage learning editores, S.A.

Deza, A. (2017) Asociatividad para la agricultura exportadora en productores de uva del distrito de San Benito. Contum azá - 2017, (Tesis), Universidad Cesar Vallejo, http://repositorio.ucv.edu.pe/bitstream/handle/UCV/11506/deza_ca.pdf?sequence=1

Flores, K. (2010). Asociatividad de los productores y cadena productiva de bananos orgánicos en el valle del río Tumbes - Perú (Tesis). Universidad de San Martin de Porres. www.administracion.usmp.edu.pe/wp-content/uploads/sites/9/.../ie_v1n1_art3.pdf

Flores, L. D. (2013). "Propuesta de asociatividad para el desarrollo económico y social de los productores de café del distrito de Kañaris - Ferreñafe" (Tesis). Facultad de Ciencias Empresariales de la Universidad Católica Santo Toribio de Mogrovejo. www.tesis.usat.edu.pe/bitstream/usat/150/1/TL_Flores_Jimenez_LuisDelfin.pdf

García, M.; Lam, F.; Núñez, M. (2014). Desarrollo de los agronegocios en américa latina y el caribe, esta publicación está disponible solamente en formato electrónico (PDF) en el sitio web institucional en http://www.iica.int

Hernández, R.; Fernández, C. \& Bautista, M. (2014) Metodología de la investigación, Mcgraw-Hill / Interamericana Editores, S.A. de C.V. sexta edición, Impreso en México.

Hernández, S. R. (2018). Metodología de la investigación las rutas de la investigación cuantitativa, cualitativa y mixta. 1ra Edición. México DF: McGraw Hill.

Jiménez, F. (2011). Crecimiento económico. Lima Perú: Fondo Editorial de la Pontificia Universidad Católica del Perú. Primera edición, impreso en tarea Asociación Grafica Educativa.

Kotler, P. \& Armstrong, G. (2012) Marketing, Pearson Educación de México, S.A. de C.V. Decimocuarta Edición, Impreso en México.

Kotler, P.; Armstrong, G. (2017). Fundamentos de marketing, Pearson educación, México, S.A. de C.V. 13 edición.

Kotler, P.; Armstrong, G. (2017). Marketing, Pearson educación, México, S.A. de C.V. 16 edición.

Maldonado, G. M. (2012) Determinantes e impactos de la asociatividad para el comercio justo: el caso de REPEBAN desde 2005 hasta el 2010 (Tesis). Pontificia Universidad Católica del Perú. http://tesis.pucp.edu.pe/repositorio/handle/123456789/1452

Moreno, J. M.; Moreno, A. (2017). Gestión y organización de la empresa agraria, editorial MUNDI PRENSA LIBROS S.A. 
Journal of the Academy Número 2 Enero-Junio 2020 - Pag. 10

Orrala, H. A. (2013) "plan de asociatividad para la asociación de agricultores ASODAGRI san marcos, de la comuna san marcos, provincia de Santa Elena, año 2013" (Tesis). Universidad Estatal Península de Santa Elena Facultad de Ciencias Administrativas Escuela de Ingeniería Comercial Carrera de Desarrollo Empresarial. La Libertad - Ecuador. http://repositorio.upse.edu.ec/bitstream/46000/1324/1

Ramos, C. (2011). Propuesta para la asociatividad de pequeños y medianos productores como estrategia de una exportación directa en el distrito de Motupe, departamento de Lambayeque - Perú. Tesis publicada. Universidad Católica Santo Toribio de Mogrovejo.

Retamozo, A. (2017) Asociatividad y crecimiento empresarial de los productores de Universidad Nacional José María Arguedas, URI: http://repositorio.unajma.edu.pe/handle/123456789/304

Robbins, S.; Coulter, M.; De Cenzo, D. (2017). Fundamentos de administración, Pearson educación, México, S.A. de C.V. cuarta edición.

Rodríguez, D. \& Riveros, H. (2016) ESQUEMAS DE COMERCIALIZACIÓN que facilitan la articulación de productores agrícolas con los mercados, Instituto Interamericano de Cooperación para la Agricultura (IICA), San José, Costa Rica. www.repiica.iica.int/docs/B4242e/B4242e.pdf.

Rodríguez, D. (2017). Institucionalidad de apoyo a la asociatividad en América Latina y el Caribe. San José, Costa Rica: Instituto Interamericano de Cooperación para la Agricultura (IICA). Creado a partir de la obra en http://www.iica.int.

Rojas, F. (2013). Formas de Asociatividad que prevalecen en la dinamización de las cadenas productivas agrícolas en Colombia (Tesis). Universidad de la Salle Facultad de Ciencias Administrativas y Contables Programa de Administración de Empresas. Bogotá, Colombia.

Http://repository.lasalle.edu.co/bitstream/handle/10185/3654/T11.13\%20R638f.pdf.

Ruiz, M. (2014) La asociatividad como estrategia para mejorar la competitividad de la Red de Productores de Quinua Sánchez Carrión, (Tesis), Universidad Nacional de Trujillo, http://dspace.unitru.edu.pe

Salcedo, S. \& Guzmán, L. (2014) Agricultura familiar en américa latina y el caribe, Publicado por la Organización de las Naciones Unidas para la Alimentación y la Agricultura Santiago, Chile; (www.fao.org/publications).

Vázquez, P. \& Zapata, J. (2015) Estudio de los factores que influyen en la asociatividad de los productores de mango del Valle Motupe - Olmos (Tesis), UNIVERSIDAD CATÓLICA SANTO TORIBIO DE MOGROVEJO. http://tesis.usat.edu.pe 